\title{
Observation of GRB afterglows with MAXI on JEM/ISS
}

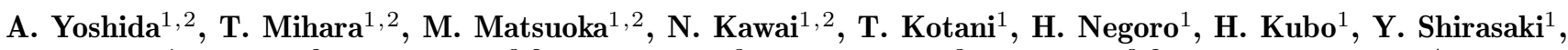 \\ B.C. Rubin ${ }^{1}$, K. Torii ${ }^{2}$, H. Tsunemi ${ }^{3,2}$, S. Kitamoto ${ }^{3}$, K. Hayashida ${ }^{3}$, E. Miyata ${ }^{3,2}$, and M. Yamauchi ${ }^{4}$ \\ 1 The Institute of Physical and Chemical Research, 2-1, Hirosawa, Wako, Saitama 351-0198, Japan \\ e-mail: ayoshida@postman.riken.go.jp \\ 2 National Space Development Agency of Japan, Tsukuba, Ibaraki 305-8505, Japan \\ 3 Osaka University, Earth and Space Science, Toyonaka, Osaka 560-0043, Japan \\ 4 Miyazaki University, Faculty of Engineering, Gakuen-kihanadai-nishi, Miyazaki 880-2155, Japan
}

Received January 21; accepted July 9, 1999

\begin{abstract}
MAXI is an X-ray all-sky-monitor which will be on the Japanese Experiment Module of the International Space Station. Its objective is to monitor X-ray sources over the entire sky with the sensitivity of less than 3 mCrab in 1 day. The GRB afterglow is one possible objective. One could expect $\sim 25$ afterglows to be detected a year at the early stage with this experiment.
\end{abstract}

Key words: gamma-rays: bursts

\section{Introduction}

The JEM (Japanese Experiment Module) is a multipurpose experiment facility for space science and technology on the International Space Station (ISS). JEM consists of a pressurized module and an Exposed Facility (EF). MAXI is the first astrophysical experiment selected for JEM-EF (Matsuoka 1997). MAXI is an X-ray allsky-monitor and its main scientific objective is to study medium-long term variations of AGNs, and to watch Galactic transients (Matsuoka et al. 1997; Kawai et al. 1997). In addition to these, its sensitivity is good enough to detect GRB afterglows in the early phase of the decay. This paper describes the MAXI experiment and discusses its expected capability of the afterglow observation.

\section{Instruments}

The FOV of MAXI slit cameras is defined by the combination of slat collimator and a slit aperture. The slat collimator defines a narrow and long field of view $\left(1.5^{\circ} \mathrm{FWHM}\right.$ $\times 90^{\circ}$ for a single collimator). The 1 -D position-sensitive $\mathrm{X}$-ray detectors are employed to map the X-ray sky within the narrow fan-beam FOV. Three collimated detectors are

Send offprint requests to: A. Yoshida combined together to cover a half circle FOV $\left(1.5^{\circ} \times 180^{\circ}\right)$ simultaneously. The ISS will rotate synchronously with its orbit so that one side will always points towards the center of the Earth and the opposite side will see the sky all the time. Figure 1 shows how it scans across the sky in the course of an orbit. Since observations are not possible at particular regions of the orbit with high charged particle flux, two fan beams are used to simultaneously cover two perpendicular fields of view, one centered on the zenith ("zenith view"), and the other centered at the forward direction of motion of the space station ("horizon view"). This will enable MAXI to achieve a more uniform coverage of the sky. To obtain the maximum scientific outputs within the constraints of the platform (JEM-EF), we designed the layout of instruments on MAXI as shown in Fig. 2. The size is $1.85 \mathrm{~m}(\mathrm{~L}) \times 0.8 \mathrm{~m}(\mathrm{~W}) \times 1.0 \mathrm{~m}(\mathrm{H})$ and the mass is about $500 \mathrm{~kg}$. It is equipped with two scientific instruments, GSC (Gas Slit Cameras) and SSC (Solid-state Slit Cameras) (Mihara et al. 1999).

GSC consists of an 1-D position sensitive proportional counter with a slat collimator and a slit on top. The proportional counter is based on the WXM/HETE2 counters with the gas of $1.4 \mathrm{~atm}$ composed of $97 \% \mathrm{Xe}$ and $3 \%$ $\mathrm{CO}_{2}$. It is sensitive to X-rays in $2-30 \mathrm{keV}$. Three identical GSC detectors are combined to cover a fan-beam field of view with $180^{\circ} \times 1.5^{\circ}$. There are four such detector sets, of which two cover the zenith view, and the other two cover the horizon view. The combined geometrical area is $\approx 6000 \mathrm{~cm}^{2}$ with the angular resolution of better than $1.5^{\circ}$ for $3-8 \mathrm{keV}$ photons. SSC uses X-ray CCD to achieve soft X-ray response. The CCD chips will be developed by Osaka University and Hamamatsu Photonics. It is the full-frame transfer type, with pixel size of $25 \mu \mathrm{m}$ square, and $1024 \times 1024$ pixels per chip. The energy range is $0.5-10 \mathrm{keV}$. Each CCD chip is employed as one-dimensional position sensitive detector with 
the fast readout. In the present design, we use 32 chips in total with an effective area of $200 \mathrm{~cm}^{2}$.

\section{Detectability of GRB afterglows}

GRB afterglows fade like $t^{-1.2}$ so one should start observation as soon as possible to obtain data with good statistics. However, the observational gap of several hours after GRB is inevitable in the observation network currently available (i.e., the WFC/BeppoSAX, BATSE/CGRO, ASM/RXTE with other telescopes for the follow-up observations). Although HETE2 can considerably shorten this period in optical/radio band, followups in X-rays will remain difficult in an inerval several minutes to a few hours after GRBs. MAXI scans the entire sky with the arc-shape FOVs. The FOV is small so that only a small number of GRBs could be caught by chance. However it could detect an afterglow if it is still brighter than the GSC's $5-\sigma$ detection limit, $F_{\mathrm{GSC}}^{\lim }$, of $7 \mathrm{mCrab}$ for one dwell (= 30 seconds) (Rubin et al. 1997) when the GSC's FOVs pass over its direction. The SSC is less sensitive than the GSC; its $5-\sigma$ detection limit is $\approx 20$ mCrab for one dwell. So, we concentrate on the GSC in the following discussions.

From the BATSE 4B catalog (Paciesas et al. 1997), the number of GRB with the Fluence in $50-300 \mathrm{keV}$ $F_{50-300}>110^{-6} \mathrm{erg} \mathrm{cm}^{-2}$ is 688 out of 1292 for the net exposure time of 2.595 years. The expected observation rate is $N_{>10^{-6}}=258.4 \mathrm{yr}^{-1}$. One could expect the X-ray flux of $>110^{-8} \mathrm{erg} \mathrm{cm}^{-2} \mathrm{~s}^{-1} \sim 500 \mathrm{mCrab}$ for these GRBs assuming a duration of $\sim 10 \mathrm{~s}$ and $10 \%$ of $\gamma$-ray energy being emitted in the X-ray band. Although the X-ray flux of afterglow scatters from burst to burst, we assume the decay $\propto t^{-1.2}$ and $(t / 10 \mathrm{~s})^{-1.2} \sim$ $\left(F_{\mathrm{GSC}}^{\lim } / 500 \mathrm{mCrab}\right)$. Then we have $t \sim 350$ seconds after the burst, in which the GSC could detect an afterglow. During $\sim 350 \mathrm{~s}$ two FOVs of the GSC can sweep $\sim 7 / 54$ of the entire sky. Since the BATSE exposure is roughly $4 \pi$, the number of afterglows expected to be de-

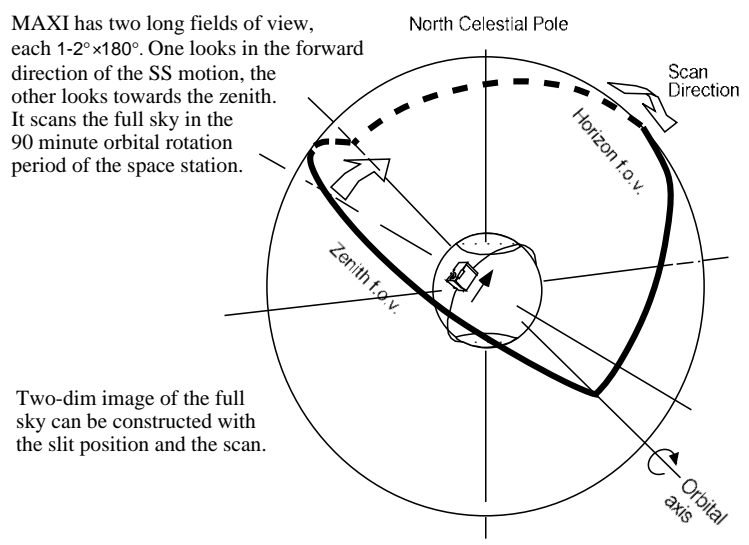

Fig. 1. The fan-beamed field of views of MAXI which scan the entire sky in one orbit

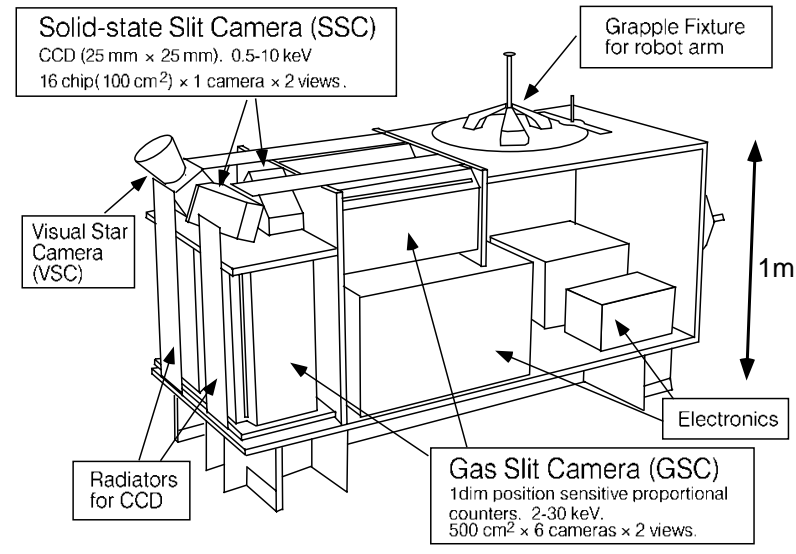

Fig. 2. A layout of the instruments

tected a year with the GSC may be estimated to be $R=N_{>10^{-6}} \cdot \varepsilon_{\mathrm{obs}} \cdot \Omega / 4 \pi$. Here $\varepsilon_{\mathrm{obs}}$, MAXI's observational efficiency, is estimated to be $\sim 0.75$ taking account of the SAA in which GSC cannot be operated due to high charged particle background. $\Omega / 4 \pi$ is an effective solid angle for the detection of afterglows, which is $\sim 7 / 54$ for $t \sim 350 \mathrm{~s}$ as mentioned above. Then one could estimate that $R \sim 25 \mathrm{yr}^{-1}$. In addtion to afterglows, GRBs themselves possibly occur in the GSC FOV of $\approx 0.16$ str. Its $5-\sigma$ detection limit is $\approx 20 \mathrm{mCrab}$ for an event with $\sim 10 \mathrm{~s}$ duration. Therefore, one may expect a detection of a burst with the fluence of $>10^{-7} \mathrm{erg} \mathrm{cm}^{-2}$. A similar estimation gives the chance detection rate of $\sim 5 \mathrm{yr}^{-1}$ for GRBs.

In conclusion, the MAXI-GSC has the capability not only to observe daily variation of weak sources such as AGNs, but also to detect GRB afterglows in the very early phase. It could bring the first opportunity to detect afterglows within about 350 seconds after GRBs.

\section{References}

Kawai N., et al., 1997, in: Proc. All-sky X-ray Observations in the Next Decade, Matsuoka M., Kawai N. (eds.), RIKEN Cosmic Radiation Lab. Special Report IPCR CR-100, p. 279

Matsuoka M., 1997, in: Proc. All-sky X-ray Observations in the Next Decade, Matsuoka M., Kawai N. (eds.), RIKEN Cosmic Radiation Lab. Special Report IPCR CR-100, p. 275

Matsuoka M., et al., 1997, SPIE Proc. 3114, p. 414

Mihara T., 1999, in: Broad Band X-ray Spectra of Cosmic Sources, 32nd COSPAR Scientific Assembly Symposium E1.1, Makishima K., Piro L. (eds.), Advances Sp. Res. (in press)

Paciesas W.S., et al., 1997, in: http://cossc.gsfc.nasa.gov/cossc/batse/4Bcatalog/

Rubin B.C., et al., 1997, in: Proc. All-sky X-ray Observations in the Next Decade, Matsuoka M., Kawai N. (eds.), RIKEN Cosmic Radiation Lab. Special Report IPCR CR-100, p. 285 